\title{
Phase tailoring of tantalum thin films deposited in deep oscillation magnetron sputtering mode
}

Fábio Ferreira $^{1,2^{*}}$, Cláudio Sousa ${ }^{1}$, Albano Cavaleiro ${ }^{1}$, André Anders $^{2}$ and João Oliveira $^{1}$

${ }^{1}$ SEG-CEMUC - Department of Mechanical Engineering, University of Coimbra, Rua Luis Reis Santos, 3030-788, Coimbra, Portugal

${ }^{2}$ Lawrence Berkeley National Laboratory, 1 Cyclotron Road, Berkeley, CA 94720, USA

*Email address: fabio.ferreira@dem.uc.pt, tel. + (351) 239790 745, fax. + (351) 239

790701

\section{Abstract}

The effect of energetic ion bombardment on the properties of tantalum thin films was investigated. To achieve such energetic ion bombardment during the process the Ta thin films were deposited by deep oscillation magnetron sputtering (DOMS), an ionized physical vapor deposition technique related to high power impulse magnetron sputtering. The peak power was between 49 and $130 \mathrm{~kW}$ and the substrate was silicon at room temperature and ground potential. The directionality and the energy of the depositing species was controlled by changing the ionization fraction of the Ta species arriving at the substrate at different peak powers. In this work, the surface morphology (AFM), microstructure (SEM), structure (XRD) and hardness and Young's modulus (nanoindentation) of the films were characterized. The ion energy distributions (IEDs) were measured using an electrostatic quadrupole ion energy and mass spectrometer (HIDEN EQP 300). The IEDs showed that the DOMS process applies a very energetic (up to $120 \mathrm{eV}$ ) ion bombardment on the growing tantalum films. Therefore, with such 
conditions it was possible to deposit pure $\alpha$-Ta (of $2 \mu \mathrm{m}$ of thickness) without the use of additional equipment, i.e., without substrate bias or substrate heating. Conditions are therefore significantly different than in previous works, offering a much simpler and cheaper solution to up-scale for industrial operation.

Keywords: Ta films, DOMS, HiPIMS, Film growth, Phase tailoring 


\section{Introduction}

Tantalum is a refractory metal with a number of unique characteristics and attractive properties such as low electrical resistivity, high melting point, and excellent chemical inertness at temperatures below $150^{\circ} \mathrm{C}[1,2]$. Due to these characteristics Ta has many applications, for instance as heat and wear resistant protective coatings, as-diffusion barriers in integrated circuits and magnetic disk drives [3-5]. Tantalum exists in two distinct phases: a stable $\alpha$-phase with a body-centered cubic lattice structure and a metastable $\beta$-phase with a tetragonal lattice structure. The tough and ductile $\alpha$-phase is required in most industrial applications, such as diffusion barrier layers, metallic corrosion protective layers, and in biomedical devices. Bulk Ta metal has the $\alpha$-phase structure while the $\beta$-phase appears in thin films. The $\beta$-phase is hard and brittle, and its presence may compromise the film performance. According to the state of the art, formation of the $\beta$-phase in magnetron sputtered films can be prevented by manipulating the deposition parameters, such as sputtering gas, energetic ion bombardment, substrate temperature and substrate material [6-10]. Among these, it was found that the use of energetic ion bombardment on the growing film played an important role in the tantalum film structure and properties [18].

In direct current magnetron sputtering (DCMS) the most influential deposition parameter with respect to ion bombardment on the growing film is the substrate bias. However, applying a negative bias to the substrate only allows us to extract process gases (Ar) ions from the plasma as the ionization degree of the sputtered material is very low (e.g., 1\%-3\%). In recent years, new magnetron sputtering deposition techniques that allow producing highly ionized fluxes of sputtered material have been developed. High peak power is applied to the target for a short period of time causing much higher plasma densities than in DCMS by ionization of the sputtered species by 
electron impact. Two of these recent developments, called High-power Impulse Magnetron Sputtering (HiPIMS) [11-14], and Modulated Pulsed Power Magnetron Sputtering (MPPMS) [15-17], were already used for the deposition of tantalum thin films. Alami et al. [18] found that HiPIMS allowed us to control the Ta phase formation and established a bias voltage window for deposition of $\alpha$-tantalum on Si. Lin et al. [19] found that all $\alpha$-tantalum films are deposited by MPPMS when the negative bias voltage was $-50 \mathrm{~V}$ or greater. In both cases the energetic bombardment of the growing film was achieved by substrate biasing. Recently, a new HIPIMS process called deep oscillation magnetron sputtering (DOMS) [20-24] was developed. This process uses large voltage oscillation packets in long modulated pulses for achieving high peak target currents and voltages. The authors have shown previously that the DOMS process allowed us to tailor the microstructure and properties of metal and nitride thin films without the need of substrate biasing [22-24]. Therefore, it is expected that the energetic ion bombardment in DOMS could also be used to tailor the phase composition and improve the structure and properties of tantalum thin films without the use of substrate bias and/or substrate heating.

In the current study, tantalum thin films were deposited on silicon by DOMS at different peak powers in order to have different levels of ion bombardment. The structure, morphology and mechanical properties of tantalum thin films were characterized. The ion energy distributions (IEDs) were measured using an electrostatic quadrupole plasma mass spectrometer (HIDEN EQP 300) both for DOMS or DCMS process. Five films were deposited by DCMS with the increase of the substrate bias for comparison purposes. 


\section{Experimental procedure}

\subsection{Film deposition}

Tantalum thin films (of $2 \mu \mathrm{m}$ of thickness) were deposited on Si (100) substrates using a continuous D.C. power source (Huttinger PFG 7500 DC) and a DOMS power supply (HiPIMS Cyprium ${ }^{\mathrm{TM}}$ plasma generator, Zpulser Inc.) at room temperature and ground potential. An example of the DOMS discharge voltage and current wave forms used in this work is shown in Figure 1 . The voltage on-time $\left(t_{\mathrm{on}}=6 \mu \mathrm{s}\right)$, oscillation period $(\mathrm{T}=50 \mu \mathrm{s})$ and pulse duration $(\mathrm{D}=1250 \mu \mathrm{s})$ were kept constant for all depositions, while the pulse frequency (F) was automatically adjusted by the DOMS power supply software in order to maintain a specified time-averaged power.

The $2 \mathrm{~cm}$ x $2 \mathrm{~cm}$ substrates were cut from $\mathrm{Si}$ (100) wafers. Prior to the depositions they were ultrasonically cleaned in a sequence of acetone and ethanol solutions baths, for $10 \mathrm{~min}$ each. They were then glued with silver glue (99.9\% purity) onto an aluminium substrate holder and placed in the deposition chamber made from high grade stainless steel with $40 \mathrm{~cm}$ x $40 \mathrm{~cm}$ x $40 \mathrm{~cm}$ dimensions. In all depositions, the substrate-to-target distance and substrate rotation was kept at $80 \mathrm{~mm}$ and $23.5 \mathrm{rpm}$, respectively. The target used consisted of $99.95 \%$ pure Ta with an area of $150 \mathrm{~mm}$ x 15 $\mathrm{mm}$ and $7 \mathrm{~mm}$ thickness. A base pressure lower than $4 \times 10^{-4} \mathrm{~Pa}$ was achieved before all depositions using a turbomolecular pump. A constant Ar (99.999\%) flow rate of 15 sccm was used in all the depositions resulting in a discharge pressure of $0.7 \mathrm{~Pa}$. A constant average target power of $1.2 \mathrm{~kW}$ was used for all depositions (DOMS and DCMS) in order to minimize variations of thermal effects during the deposition of the films. The deposition time was changed in order to achieve $2 \mu \mathrm{m}$ of thickness.

The tantalum thin films were deposited by DOMS with the increase of the peak power $\left(\mathrm{P}_{\mathrm{p}}\right)$ in order to increase the ionization of the sputtered material during the 
process. The $\mathrm{P}_{\mathrm{p}}$ was varied between 49 and $130 \mathrm{~kW}$ by changing the charging voltage $\left(D_{i n t}\right)$ between 270 and $400 \mathrm{~V}$. The peak power $\left(P_{p}\right)$ is defined as the product $V_{p} \times I_{p}$. The main DOMS deposition parameters are compiled in table 1. Five films were deposited by DCMS with increased negative substrate bias $(-30 \mathrm{~V},-50 \mathrm{~V},-80 \mathrm{~V}$ and $120 \mathrm{~V}$ ) for comparison purposes. In these depositions the voltage target and current target were $333 \mathrm{~V}$ and $3.75 \mathrm{~A}$, respectively.

\subsection{Ion energy distribution measurements}

The ion energy distributions (IEDs) were measured using an electrostatic quadrupole plasma mass spectrometer (HIDEN EQP 300). This equipment can measure up to $1 \mathrm{keV} / \mathrm{charge}$ and is assisted by $70 \mathrm{l} / \mathrm{s}$ turbo pump for differential pumping of the instrument. The tantalum target with a diameter of $7.6 \mathrm{~cm}$ was mounted in front of the EQP orifice (100 $\mu \mathrm{m}$ diameter) with a $10 \mathrm{~cm}$ distance between them. An average target power of $600 \mathrm{~W}$ was used for both DOMS and DCMS. The IEDs were measured from 0 to $100 \mathrm{~V}$ scan voltage with a step size of $0.5 \mathrm{~V}$ and a $1000 \mathrm{~ms}$ dwell time. The extractor voltage used in EQP measurement was equal to $40 \mathrm{~V}$. In accordance with the reference [41], after the measurements the raw data were corrected for the double charge ions by multiplying the scan voltage by two and dividing the count rate by two to account for the energy bin width. The following ions were analysed: $\mathrm{Ta}^{+}, \mathrm{Ta}^{2+}, \mathrm{Ar}^{+}, \mathrm{Ar}^{2+}$. It has to be mentioned that the here-presented distributions merely provide a semi-quantitative description of the energy distributions involved during the DOMS tantalum sputtering process because difficult-to-assess instrument functions such as the acceptance angle distort the measured functions. 


\subsection{Film characterization}

The crystal structure of tantalum thin films was analysed by X-ray diffraction (XRD) (PANalytical X'Pert PRO MPD) using $\mathrm{Cu} \mathrm{K} \alpha$ radiation (45 kV and $40 \mathrm{~mA}$ ) with a parallel beam in $\theta-2 \theta$ geometry. The incident beam optics consisted of a hybrid monochromator (with a $\mathrm{Cu} \mathrm{W/Si} \mathrm{mirror} \mathrm{and} \mathrm{a} \mathrm{double} \mathrm{crystal} \mathrm{Ge} \mathrm{(220)).} \mathrm{A} \mathrm{parallel} \mathrm{plate}$ collimator $\left(0.7^{\circ}\right)$ and Soller slits $\left(0.004^{\circ}\right)$ were mounted on the path of the diffracted beam. A PIXcel detector in receiving slit mode was used for X-ray collection. The thickness and morphology of the films were studied by scanning electron microscopy (SEM) using a Quanta 400FEG ESEM. The SEM images obtained for the cross section and surface area in this work were achieved with a $2 \mathrm{keV}$ beam. The film topography and roughness were examined by atomic force microscopy (AFM) using a Bruker Innova equipment in contact mode. Images with a surface area of $2 \mu \mathrm{m} \times 2 \mu \mathrm{m}$ resolution. The hardness of the films was measured by nano-indentation (MicroMaterialsNano Tester) using a Berkovich diamond indenter. The hardness was evaluated from load-displacement curves using the depth-sensing method. Hardness measurements were done with $10 \mathrm{mN}$ loads in order to ensure an indentation depth less than $10 \%$ of the coating's thickness. 16 hardness measurements were performed on each specimen. 


\section{Results}

\subsection{Ion energy distribution functions}

The time-averaged ion energy distributions (IEDFs) for ${ }^{181} \mathrm{Ta}^{+},{ }^{181} \mathrm{Ta}^{2+},{ }^{40} \mathrm{Ar}^{+}$and ${ }^{40} \mathrm{Ar}^{2+}$ ion species measured from the DOMS plasma obtained at different peak powers $(37,52,84$ and $114 \mathrm{~kW})$ during Ta sputtering are displayed in figure 2 . The ion energy distributions obtained from the DCMS plasma are also displayed in figure 2 for comparison. All the regimes reveal a low energy peak for $\mathrm{Ar}^{+}$centred near $5 \mathrm{eV}$ with a maximum energy close to $8 \mathrm{eV}$ (figure 2a). This peak corresponds to thermalized $\mathrm{Ar}^{+}$ ions after being accelerated over the sheath from the plasma to the substrate. The energetic position of the peak corresponds to the plasma potential. A similar IEDF for $\mathrm{Ar}^{+}$was found by Lin et al. [19] for a MPPMS discharge. The $\mathrm{Ar}^{2+}$ IEDs (figure 2b) exhibit a low energy peak centred close to $9 \mathrm{eV}$ for both processes although the number of ions is almost one order of magnitude higher in DOMS than in DCMS. Once again, this peak corresponds to thermalized ions accelerated over the substrate (or detector entrance) sheath. However, the IEDFs of the DOMS process exhibit an additional high energy tail with increased maximum energy (from 30 to $48 \mathrm{eV}$ ) with increasing peak power. Hecimovic et al. [28] found that the $\mathrm{Ar}^{2+}$ IEDF in their HiPIMS discharge with a chromium target consisted of high energy ions (with maximum energy up to $25 \mathrm{eV}$ ) during the HiPIMS pulse while only a low energy $\mathrm{Ar}^{2+}$ peak was detected in postdischarge conditions. The authors proposed that the high energy $\mathrm{Ar}^{2+}$ ions were probably created through collisions with high energy Cr ions. Momentum transfer through collisions with the highly energetic Ta species also explains the high energy $\mathrm{Ar}^{2+}$ ions generated within the DOMS discharge. Note, however, that the energy of the 
vast majority of the $\mathrm{Ar}^{2+}$ ions is within the low energy peak and thus that energetic $\mathrm{Ar}^{2+}$ ions only have small influence on the film growth.

The $\mathrm{Ta}^{+}$IEDF in DCMS also show a low energy centred close to $6 \mathrm{eV}$ but this peak extends up to $20 \mathrm{eV}$ (figure 2c), i.e., up to higher energy than in DCMS. The higher energy $\mathrm{Ta}^{+}$ions result from the original Thompson distribution function of the sputter-ejected atoms [29]. Much broader $\mathrm{Ta}^{+}$IEDFs are found in DOMS. The maximum energy of the $\mathrm{Ta}^{+}$ions reaches $65 \mathrm{eV}$ regardless of peak power. The DOMS discharge produces highly energetic single charged Ta ions with much higher energies than those generated in DCMS and MPPMS discharges [19]. This result is at least partly due to the larger cathode sheath voltage which develops in DOMS as the higher energy of the $\mathrm{Ar}^{+}$ions bombarding the target will translate to higher average energy of the sputtered Ta neutrals. However, the origin of the energetic metal species generated by HiPIMS is still an open issue [30]. Recently, Anders et al. proposed that ionization zones in HIPIMS are locations of locally enhanced potential, incorporating double layers (DL) which result in potential humps that could explain the relatively high energy of the ions at the substrate [31]. Maszl et al. [30] also concluded that energetic $\mathrm{Ti}^{+}$ions are created in the spokes region and subsequently accelerated inside the DL surrounding the spoke. A similar mechanism could also explained the highly energetic Ta ions produced by the DOMS discharges generated in this work. Note however that the total count of $\mathrm{Ta}^{+}$ions decreases with increasing peak power. Although the fraction of ionized sputtered metal species increases with increasing peak power [14], the total number of metal ions reaching the substrate can decrease due to several effects, such as back-attraction of ionized metal species and the yield effect, that also cause a deposition rate decrease with increasing peak power [38]. The Ta ${ }^{2+}$ IEDF in DCMS (figure 2d) exhibit a peak energy at $8 \mathrm{eV}$ and a tail extending up to $25 \mathrm{eV}$. The amount of $\mathrm{Ta}^{2+}$ ions 
is much lower than $\mathrm{Ta}^{+}$ions while the more pronounced low energy peak shows that the $\mathrm{Ta}^{2+}$ are either more efficiently thermalized or generated outside accelerating field structures than the $\mathrm{Ta}^{+}$ions. In the DOMS discharge the $\mathrm{Ta}^{2+}$ IEDF exhibits a peak energy close to $10 \mathrm{eV}$ and a tail extending to even higher energy than for the $\mathrm{Ta}^{+}$ions, i.e., reaching between 95 and $120 \mathrm{eV}$ depending on the applied peak power.

Furthermore, the ions count in the high-energy portion of the $\mathrm{Ta}^{2+}$ IEDF increases with increasing peak power.

The metal IEDFs $\left(\mathrm{Ta}^{+}\right.$and $\left.\mathrm{Ta}^{2+}\right)$ in DOMS exhibit much larger high energy tails than the process gas IDEFs $\left(\mathrm{Ar}^{+}\right.$and $\left.\mathrm{Ar}^{2+}\right)$ which suggest that a large fraction of the ion species within the plasma are energetic Ta ions. During a DOMS deposition the growing Ta film will be under intense and energetic bombardment of Ta ions while in DCMS most of the ions reaching the substrate are process gas ions with much lower energy.

\subsection{Direct Current Magnetron Sputtering (DCMS)}

Figure 3 shows the X-ray diffraction patterns (between $30^{\circ}$ and $45^{\circ}$ ) of Ta thin films deposited by DCMS with increasing substrate bias $(0,-30,-50,-80$ and $-120 \mathrm{~V})$. The X-ray diffractograms exhibit $\beta$-Ta (002) peaks for all bias values. These results agree well with previous works $[18,25,26]$. The $\beta$-Ta (002) peak is shifted to higher diffraction angles (to smaller lattice spacing) as compared to the corresponding diffraction pattern standard, which is most probably due to the development of tensile stresses in the films. The peak shift decreases with increasing peak power and at the highest bias (-120 V) the $\beta$-Ta (002) peak is close to the unstressed position. This result is consistent with the increased energy of the Ar ions, which dominated DCMS as 
shown in the corresponding IEDF, as the bias is negatively increased. Despite of the increased energy of the ions bombarding the film, similar sharp $\beta$-Ta (002) peaks are observed irrespectively of the deposition bias. Dalla Torre et al. [32] have shown the importance of a mass match during growth of tantalum thin films by sputtering. The lower mass Ar ions as compared to the film-forming species are rather ineffective at influencing the Ta film structure.

Selected SEM micrographs of the Ta thin films deposited by DCMS with increase of substrate bias are shown in figure 4 . All the films have a columnar morphology and exhibit brittle fractures typical of the $\beta$-phase. Similar Brittle fractures were also observed by Myers et al. [33] and corresponds to the T zone in Thornton's structure zone diagram [34]. The Ta thin films deposited with -30 and $-80 \mathrm{~V}$ exhibit a surface with three-sided pyramidal structures. Increasing the substrate bias to $-120 \mathrm{~V}$ resulted in the formation of structure with ellipsoidal morphology.

\subsection{Deep Oscillation Magnetron Sputtering (DOMS)}

The deposition rate of the tantalum thin films deposited by DOMS is shown in figure 5. The deposition rate was calculated by dividing the film thickness (measured by SEM) by the deposition time. An increase of the peak power by a factor of almost three results in a loss of $43 \%$ in deposition rate. The loss of deposition rate in DOMS [2224], and more generally in HIPIMS [35-39], as compared to DCMS, is well documented in the literature. The deposition rate decreases with increasing peak power in HIPIMS due to the back-attraction of some of the ionized sputtered metal species to the target, the so-called return effect. On the other hand, the yield effect results from the lower efficiency of the sputtering process at the typically higher process voltages in HIPIMS, 
as compared to DCMS, which originates in the sub-linear energy dependence of the sputtering yield [38, 39]. Among other effects, Panjan et al. and Franz et al. also reported an increased flux of the sputtered species sideways, i.e., to higher angles with respect to target surface normal in HIPIMS than in DCMS [40, 41].

Figure 6 shows the X-ray diffraction patterns (between $2 \theta=30^{\circ}$ and $45^{\circ}$ ) of $\mathrm{Ta}$ thin films deposited by DOMS with increase of peak power $\left(\mathrm{P}_{\mathrm{p}}\right)$. All the diffractograms of Ta films deposited with a peak power up to $105 \mathrm{~kW}$ only exhibit the $\alpha$-Ta (110) diffraction peak showing that pure $\alpha$-Ta was deposited. In all the diffractograms the $\alpha$ Ta (110) diffraction angle is lower than in the corresponding diffraction pattern standard, which indicates the formation of compressive stresses. However, the shift of the $\alpha$-Ta (110) peak to higher diffraction angles with increasing peak power shows that the compressive stress is partially relaxed at higher peak power. The $\alpha$-Ta (110) diffraction peaks are broader than in DCMS indicating smaller grain sizes. Ren et al. proposed that small grain size of the $\alpha$-Ta may be due to the high density of nucleation sites on the surface which is bombarded with energetic ions [42]. Increasing the peak power to $130 \mathrm{~kW}$ results in the deposition of mixed $\alpha$ - and $\beta$-Ta phases as confirmed by the presence of the corresponding diffraction peaks. However, the diffraction peaks are much broader and much less intense than at lower $\mathrm{P}_{\mathrm{p}}$, while the $\beta$-Ta (200) peak position coincides with the value in the corresponding diffraction pattern standard.

The cross-section and surface morphology of Ta thin films deposited by DOMS has been observed by SEM (Figure 7). In cross-section all Ta thin films exhibit a columnar morphology with closely-packed columns and compact grain boundaries. This morphology corresponds to the T zone in the generalized structure zone diagram [43], and it was also observed in previous works with the use of DOMS power supply [22, 23]. The Ta thin film deposited with $130 \mathrm{~kW}$ reveal two different types of fracture, the 
lower part of film (nearby the substrate) exhibits a brittle fracture while the upper part exhibits a ductile fracture. Myers et al. observed the same behaviour and attributed these different fractures types to the presence of different Ta phases [33]. This is in agreement with the X-ray diffractions which revealed a mixed phase structure. The surface of the film deposited with a peak power of $49 \mathrm{~kW}$ displays dense, regularly spaced grain facets similar to the ones reported by Ren et al. for $\alpha$-Ta [42]. Increasing the peak power results in similar but lower sized features on the film surface, even for the film deposited at $130 \mathrm{~kW}$ as $\alpha$-Ta was deposited on the upper part of the film.

Figure 8 shows the AFM 3D topographic images of Ta thin films of different $P_{p}$, deposited by DOMS. The AFM measurements were done in contact mode over $2 \times 2 \mu \mathrm{m}$ area. These analyses confirm what was observed in the SEM analyses. In AFM is also observed the surface structures downsize with the increase of the peak power. All the AFM images confirm the deposition of Ta films without significant defects.

The average roughness (measured by AFM) of Ta thin films deposited with increasing peak power is displayed in figure 9. The increase of peak power, i.e., increase of ion bombardment, results in a decrease of the roughness in two steps. This result agrees well with the decrease of the surfaces structures observed in SEM and AFM analyses.

Figure 10 shows the hardness and Young's modulus of the DOMS Ta thin films deposited at different peak powers. The hardness values reported in the literature for $\alpha$-Ta are between 8 and $12 \mathrm{GPa}$ while higher hardness is reported for $\beta$-Ta (16 to $20 \mathrm{GPa}$ ) depending on the deposition method [44]. All the Ta thin films deposited between 49 and $105 \mathrm{~kW}$ have close to $11.5 \mathrm{GPa}$. All these films have pure $\alpha$-Ta phase, therefore, this result agrees well with the hardness values mention above. The Ta thin film deposited with a peak power of $130 \mathrm{~kW}$ reveals a slightly higher hardness $(12.7$ 
GPa) which agrees well with the deposition of $\beta$-Ta mixed with $\alpha-T a$. The Young's modulus values of the Ta films range between 210 and $220 \mathrm{GPa}$. 


\section{Discussion}

All the films deposited by DCMS consist only of the $\beta$-Ta phase and clearly have columnar microstructures. Increasing the bias voltage did not significantly change the films structure and microstructure although bias voltages as high as $-120 \mathrm{~V}$ were used in this work. As was shown in section 3.1, the DCMS discharge consist mainly of process gas ions $\left(\mathrm{Ar}^{+}\right)$which are extracted from the plasma and bombard the growing film after acceleration in the substrate sheath. Although very high energy ions were used during deposition at the highest bias, the momentum transfer to the growing film is rather ineffective as it is an indirect process which relies on collisions of the accelerated Ar ions with the Ta ad-atoms. Moreover, the mass mismatch between Ar and Ta further reduces the momentum transfer effectiveness. As a result the Ta thin films consist only of the $\beta$-Ta phase and even develop tensile stresses irrespective of the bias voltage. The deposition of pure $\alpha$-Ta thin films by sputtering has been demonstrated by Ino et al. [6]. Pure $\alpha$-Ta deposition was achieved at low bias voltages (between -5 and $-25 \mathrm{~V}$ ) but only at high $\mathrm{Ar}^{+}$fluxes which were achieved by using additional dual rf excitation of the plasma. In this work no additional plasma excitation sources were used in the DCMS depositions and the $\mathrm{Ar}^{+}$flux impinging on the growing film is insufficient to reach the pure $\alpha$-Ta deposition region in the experimental phase formation diagram proposed by Ino et al. [6].

The Ta thin films deposited by DOMS up to $105 \mathrm{~kW}$ consist only of the $\alpha$-Ta phase. Compressive stress are formed while the grain size is smaller than in the DCMS films. On the other hand, increasing the peak power leads to the deposition of more compact microstructures with much lower surface roughness. As was shown in section 3.1, the DOMS plasma is dominated by highly energetic $\mathrm{Ta}^{+}$and $\mathrm{Ta}^{2+}$ ions which bombard the substrate during film grow. The momentum transfer to the film is then much more 
effective than in DCMS as it is directly carried out by the film-forming species. As a result, pure $\alpha$-Ta is deposited while the films microstructure evolves according to the peak power, i. e., according to the energy and flux of the bombarding ions. The DOMS process allows us to deposit pure $\alpha$-Ta without the addition of supplementary equipment, without substrate biasing and without substrate heating in contrast to previous works $[3,19,42]$. However, the energetic bombardment induced the formation of compressive stresses in the films as also found in previous works $[3,6]$.

Lin et al. performed IEDFs measurements of $\mathrm{Ar}^{+}, \mathrm{Ta}^{+}$, and $\mathrm{Ta}^{2+}$ in a MPPMS discharge over a Ta target [19]. The IEFDs were shown to consist only in a low energy peak centred around $3 \mathrm{eV}$ and with maximum energy of $12 \mathrm{eV}$ for $\mathrm{Ar}^{+}$and $\mathrm{Ta}^{+}$and slightly lower $(8 \mathrm{eV})$ for $\mathrm{Ta}^{2+}$ (however, no mention is made in this work about the charge state correction of energy, which, if it was omitted, would put the $\mathrm{Ta}^{2+}$ peak at $16 \mathrm{eV}$ ). In this work the IEDFs of the metallic ions also display a similar low energy peak, corresponding to thermalized species, but in all cases an additional high energy tail, extending up to $48 \mathrm{eV}$ for $\mathrm{Ta}^{+}$and up to $120 \mathrm{eV}$ for $\mathrm{Ta}^{2+}$, is also detected. The negative target voltages are higher in the DOMS process (between -909 and $-1296 \mathrm{~V}$, table 1) than in the MPPMS process used by Lin et al. (below $-500 \mathrm{~V}$ ). Thus, the $\mathrm{Ar}^{+}$ions in DOMS gain more energy upon acceleration in the target sheath, and, although some of this energy will contribute to an increase of the sputtering yield this will also contribute to an increased energy of the sputtered species. As a result more energetic metallic ions will reach the substrate in DOMS as compared to MPPMS.

The XRD analyses shows that a mixed $\alpha$ - and $\beta$-Ta film was deposited by DOMS at the highest peak power $(130 \mathrm{~kW})$. Accordingly, the hardness of this film is higher than that of the pure $\alpha$-Ta films deposited by DOMS at lower peak power. The SEM cross section of the film clearly shows that the film consists of $\beta$-Ta in the lower 
part while $\alpha$-Ta is grown in the upper part. Alami et al. [18] reported on an energy window for the deposition of pure $\alpha$-Ta by HIPIMS. The authors concluded that the window's higher energy limit was due to relaxation of the film stress under energetic ion bombardment. On the other hand, Ino et al. [6] found that the $\beta$-Ta phase is always formed at higher energies, i. e., when higher bias voltages (above $25 \mathrm{~V}$ ) are used to accelerate the argon ions impinging on the growing film. The authors concluded that $\beta$ Ta formation was caused by the defects induced by the recoil implantation of Ta atoms in the excessive high-energy ion bombardment conditions. Both explanations cited above for the formation of the $\beta$-Ta phase under energetic bombardment during deposition are consistent with the results obtained in this work. A relaxation of the film stress with increasing peak power was observed by X-ray diffraction while the more compact microstructures and much lower roughness of the films deposited with increasing peak power could be explained by re-nucleation due to increasing defects formation, morphology that corresponds to the zone 3 in the generalized structure zone diagram [43].

\section{Conclusions}

The IEDFs measurement of the DCMS and DOMS discharges using a tantalum target revealed very different plasma environments for each deposition process. The DCMS plasma is dominated by low energy process gas ions while the DOMS discharge contains a large fraction of highly energetic (up to $120 \mathrm{eV}$ ) Ta ions. Although substrate biases up to $-120 \mathrm{~V}$ were used in DCMS, all the deposited films consist in pure $\beta$-Ta. This result was attributed to the ineffective indirect momentum transfer from the $\mathrm{Ar}$ ions to the growing film that results in insufficient energy supply to grow the $\alpha$-Ta phase. In contrast, $\alpha$-Ta was formed in all films deposited by DOMS without the need of 
substrate biasing (samples at ground potential) and/or substrate heating. The momentum transfer to the film is much more effective than in DCMS as it is directly carried out by the film forming species. As a result, the films microstructure evolves according to the peak power, i. e., according to the energy and flux of the bombarding ions. The DOMS process allowed us to deposit pure $\alpha-$ Ta without the addition of supplementary equipment and without the need of substrate biasing as reported in previous works. 


\section{Acknowledgments}

This research is sponsored by FLAD, FEDER funds through the program COMPETE - Programa Operacional Factores de Competitividade - and by national funds through FCT - Fundação para a Ciência e a Tecnologia -, under the projects: PTDC/EME-TME/122116/2010, PTDC/EMS-TEC/1805/2012 and PEstC/EME/UI0285/2013, as well as the grant (SFRH/BD/68740/2010). Work at Lawrence Berkeley National Laboratory is supported by the U.S. Department of Energy under Contract No. DE-AC02-05CH11231. 


\section{References}

[1] L. Gladczuk, A. Patel, C. S. Paur, and M. Sosnowski, "Tantalum films for protective coatings of steel," Thin Solid Films, vol. 467, pp. 150-157, 2004.

[2] M. Grosser and U. Schmid, "The impact of sputter conditions on the microstructure and on the resistivity of tantalum thin films," Thin Solid Films, vol. 517, pp. 4493-4496, 2009.

[3] S. Maeng, L. Axe, T. Tyson, and P. Cote, "Corrosion behaviour of electrodeposited and sputtered Cr coatings and sputtered Ta coatings with $\alpha$ and $\beta$ phases," Surface and Coatings Technology, vol. 200, pp. 5767-5777, 2006.

[4] L. Liu, H. Gong, Y. Wang, J. Wang, A. Wee, and R. Liu, "Annealing effects of tantalum thin films sputtered on [001] silicon substrate," Materials Science and Engineering: C, vol. 16, pp. 85-89, 2001.

[5] K. Holloway and P. M. Fryer, "Tantalum as a diffusion barrier between copper and silicon," Applied Physics Letters, vol. 57, pp. 1736-1738, 1990.

[6] K. Ino, T. Shinohara, T. Ushiki, and T. Ohmi, "Ion energy, ion flux, and ion species effects on crystallographic and electrical properties of sputter-deposited Ta thin films," Journal of Vacuum Science \& Technology A, vol. 15, pp. 2627-2635, 1997.

[7] D. W. Matson, E. D. McClanahan, J. P. Rice, S. L. Lee, and D. Windover, "Effect of sputtering parameters on Ta coatings for gun bore applications," Surface and Coatings Technology, vol. 133, pp. 411-416, 2000.

[8] N. Schwartz and E. Feit, "Impurity Effects in the Nucleation of Alpha (bcc)-Tantalum or Beta-Tantalum Films," Journal of The Electrochemical Society, vol. 124, pp. 123-131, 1977.

[9] G. Chen, P. Lee, and S. Chen, "Phase formation behavior and diffusion barrier property of reactively sputtered tantalum-based thin films used in semiconductor metallization," Thin Solid Films, vol. 353, pp. 264-273, 1999. 
[10] L. Hallmann and P. Ulmer, "Effect of sputtering parameters and substrate composition on the structure of tantalum thin films," Applied Surface Science, vol. 282, pp. 1-6, 2013.

[11] V. Kouznetsov, K. Macak, J. M. Schneider, U. Helmersson, and I. Petrov, "A novel pulsed magnetron sputter technique utilizing very high target power densities," Surface and coatings technology, vol. 122, pp. 290-293, 1999.

[12] U. Helmersson, M. Lattemann, J. Bohlmark, A. P. Ehiasarian, and J. T. Gudmundsson, "Ionized physical vapor deposition (IPVD): A review of technology and applications," Thin Solid Films, vol. 513, pp. 1-24, 2006.

[13] D. Lundin and K. Sarakinos, "An introduction to thin film processing using high-power impulse magnetron sputtering," Journal of Materials Research, vol. 27, pp. 780-792, 2012.

[14] A. Ehiasarian, R. New, W.-D. Münz, L. Hultman, U. Helmersson, and V. Kouznetsov, "Influence of high power densities on the composition of pulsed magnetron plasmas," Vacuum, vol. 65, pp. 147-154, 2002.

[15] J. Lin, W. D. Sproul, J. J. Moore, Z. Wu, S. Lee, R. Chistyakov, et al., "Recent advances in modulated pulsed power magnetron sputtering for surface engineering," Jom, vol. 63, pp. 48-58, 2011.

[16] J. Lin, J. J. Moore, W. D. Sproul, B. Mishra, and Z. Wu, "Modulated pulse power sputtered chromium coatings," Thin Solid Films, vol. 518, pp. 1566-1570, 2009.

[17] J. Lin, J. Moore, W. Sproul, B. Mishra, J. Rees, Z. Wu, et al., "Ion energy and mass distributions of the plasma during modulated pulse power magnetron sputtering," Surface and Coatings Technology, vol. 203, pp. 3676-3685, 2009.

[18] J. Alami, P. Eklund, J. M. Andersson, M. Lattemann, E. Wallin, J. Bohlmark, et al., "Phase tailoring of Ta thin films by highly ionized pulsed magnetron sputtering," Thin Solid Films, vol. 515, pp. 3434-3438, 2007.

[19] J. Lin, J. J. Moore, W. D. Sproul, S. L. Lee, and J. Wang, "Effect of negative substrate bias on the structure and properties of Ta coatings deposited using modulated pulse power magnetron sputtering," IEEE transactions on plasma science, vol. 38, pp. 3071-3078, 2010. 
[20] J. Lin, B. Wang, W. D. Sproul, Y. Ou, and I. Dahan, "Anatase and rutile TiO2 films deposited by arc-free deep oscillation magnetron sputtering," Journal of Physics D: Applied Physics, vol. 46, p. 084008, 2013.

[21] J. Lin and W. D. Sproul, "Structure and properties of Cr 2 O 3 coatings deposited using DCMS, PDCMS, and DOMS," Surface and Coatings Technology, vol. 276, pp. 70-76, 2015.

[22] F. Ferreira, J. Oliveira, and A. Cavaleiro, "CrN thin films deposited by HiPIMS in DOMS mode," Surface and Coatings Technology, vol. 291, pp. 365-375, 2016.

[23] F. Ferreira, R. Serra, J. Oliveira, and A. Cavaleiro, "Effect of peak target power on the properties of $\mathrm{Cr}$ thin films sputtered by HiPIMS in deep oscillation magnetron sputtering (DOMS) mode," Surface and Coatings Technology, vol. 258, pp. 249-256, 2014.

[24] J. Oliveira, F. Fernandes, F. Ferreira, and A. Cavaleiro, "Tailoring the nanostructure of $\mathrm{Ti}-\mathrm{Si}-\mathrm{N}$ thin films by HiPIMS in deep oscillation magnetron sputtering (DOMS) mode," Surface and Coatings Technology, vol. 264, pp. 140-149, 2015.

[25] A. Jiang, T. A. Tyson, L. Axe, L. Gladczuk, M. Sosnowski, and P. Cote, "The structure and stability of $\beta$-Ta thin films," Thin solid films, vol. 479, pp. 166-173, 2005.

[26] H. Högberg, L. Tengdelius, M. Samuelsson, J. Jensen, and L. Hultman, " $\beta-T a$ and $\alpha-\mathrm{Cr}$ thin films deposited by high power impulse magnetron sputtering and direct current magnetron sputtering in hydrogen containing plasmas," Physica B: Condensed Matter, vol. 439, pp. 3-8, 2014.

[27] P. Scherrer, "Zsigmondy’s Kolloidchemie," Nachrichten der Göttinger Gesellschaft, vol. 98, p. 394, 1918.

[28] A. Hecimovic and A. P. Ehiasarian, "Spatial and temporal evolution of ion energies in high power impulse magnetron sputtering plasma discharge," Journal of Applied Physics, vol. 108, p. 063301, 2010. 
[29] M. Thompson, "II. The energy spectrum of ejected atoms during the high energy sputtering of gold," Philosophical Magazine, vol. 18, pp. 377-414, 1968.

[30] C. Maszl, W. Breilmann, J. Benedikt, and A. von Keudell, "Origin of the energetic ions at the substrate generated during high power pulsed magnetron sputtering of titanium," Journal of Physics D: Applied Physics, vol. 47, p. 224002, 2014.

[31] A. Anders, M. Panjan, R. Franz, J. Andersson, and P. Ni, "Drifting potential humps in ionization zones: the "propeller blades" of high power impulse magnetron sputtering," Applied physics letters, vol. 103, p. 144103, 2013.

[32] J. Dalla Torre, G. Gilmer, D. Windt, R. Kalyanaraman, F. Baumann, P. O'Sullivan, et al., "Microstructure of thin tantalum films sputtered onto inclined substrates: Experiments and atomistic simulations," Journal of applied physics, vol. 94, pp. 263-271, 2003.

[33] S. Myers, J. Lin, R. M. Souza, W. D. Sproul, and J. J. Moore, "The $\beta$ to $\alpha$ phase transition of tantalum coatings deposited by modulated pulsed power magnetron sputtering," Surface and Coatings Technology, vol. 214, pp. 38-45, 2013.

[34] J. A. Thornton, "Influence of apparatus geometry and deposition conditions on the structure and topography of thick sputtered coatings," Journal of Vacuum Science \& Technology, vol. 11, pp. 666-670, 1974.

[35] M. Samuelsson, D. Lundin, J. Jensen, M. A. Raadu, J. T. Gudmundsson, and U. Helmersson, "On the film density using high power impulse magnetron sputtering," Surface and Coatings Technology, vol. 205, pp. 591-596, 2010.

[36] J. Alami, K. Sarakinos, G. Mark, and M. Wuttig, "On the deposition rate in a high power pulsed magnetron sputtering discharge," Applied Physics Letters, vol. 89, p. 154104, 2006.

[37] D. Christie, "Fundamentals of high power pulsed magnetron sputtering: Visualization of mechanisms for rate reduction and increased ion fraction," Czechoslovak Journal of Physics, vol. 56, pp. B93-B97, 2006.

[38] A. Anders, "Deposition rates of high power impulse magnetron sputtering: physics and economics," Journal of Vacuum Science \& Technology A, vol. 28, pp. 783790, 2010. 
[39] J. Emmerlich, S. Mráz, R. Snyders, K. Jiang, and J. M. Schneider, "The physical reason for the apparently low deposition rate during high-power pulsed magnetron sputtering," Vacuum, vol. 82, pp. 867-870, 2008.

[40] M. Panjan, R. Franz, and A. Anders, "Asymmetric particle fluxes from drifting ionization zones in sputtering magnetrons," Plasma Sources Science and Technology, vol. 23, p. 025007, 2014.

[41] R. Franz, C. Clavero, J. Kolbeck, and A. Anders, "Influence of ionisation zone motion in high power impulse magnetron sputtering on angular ion flux and $\mathrm{NbO} x$ film growth," Plasma Sources Science and Technology, vol. 25, p. 015022, 2016.

[42] H. Ren and M. Sosnowski, "Tantalum thin films deposited by ion assisted magnetron sputtering," Thin Solid Films, vol. 516, pp. 1898-1905, 2008.

[43] A. Anders, "A structure zone diagram including plasma-based deposition and ion etching," Thin Solid Films, vol. 518, pp. 4087-4090, 2010.

[44] S. Lee, M. Doxbeck, J. Mueller, M. Cipollo, and P. Cote, "Texture, structure and phase transformation in sputter beta tantalum coating," Surface and Coatings Technology, vol. 177, pp. 44-51, 2004. 
Table 1 - Deposition conditions used to deposit tantalum thin films by DOMS.

\begin{tabular}{cccccc}
\hline $\begin{array}{c}\mathrm{DC}_{\text {int }} \\
(\mathrm{V})\end{array}$ & $\begin{array}{c}\mathrm{V}_{\mathrm{p}} \\
(\mathrm{V})\end{array}$ & $\begin{array}{c}\mathrm{I}_{\mathrm{p}} \\
(\mathrm{A})\end{array}$ & $\begin{array}{c}\mathrm{P}_{\mathrm{p}} \\
(\mathrm{kW})\end{array}$ & $\begin{array}{c}\mathrm{F}_{\mathrm{i}} \\
(\mathrm{Hz})\end{array}$ & $\begin{array}{c}\text { Dep. time } \\
(\text { min. })\end{array}$ \\
\hline 270 & 909 & 54 & 49 & 271 & 80 \\
300 & 987 & 66 & 66 & 219 & 8 \\
330 & 1084 & 76 & 82 & 180 & 95 \\
360 & 1172 & 90 & 105 & 148 & 105 \\
400 & 1296 & 101 & 130 & 120 & 115 \\
\hline
\end{tabular}




\section{Figure captions}

Figure $1-$ a) The target voltage and current oscillation waveforms measured during the Ta thin film depositions. b) Small oscillation pulses within one long pulse.

Figure 2 - Ion energy distributions (IEDs) of a) ${ }^{40} \mathrm{Ar}^{+}$; b) ${ }^{40} \mathrm{Ar}^{2+}$; c) ${ }^{181} \mathrm{Ta}^{+}$and d) ${ }^{181} \mathrm{Ta}^{2+}$ species measured from DOMS plasma with different peak powers during sputtering a Ta target in pure Ar atmosphere. DCMS IEDs are also plotted for comparison.

Figure 3 - X-ray diffraction patterns of the films prepared by DCMS with the increase of substrate bias.

Figure 4 - SEM micrographs of Ta $2 \mu \mathrm{m}$ thick films deposited by DCMS with substrate bias of a, b) $-30 \mathrm{~V}$; c, d) $-80 \mathrm{~V}$; e, f) $-120 \mathrm{~V}$.

Figure 5 - Deposition rate of Ta thin films deposited by DOMS as function of $\mathrm{P}_{\mathrm{p}}$.

Figure 6 - XRD patterns of the films deposited by DOMS as function of $\mathrm{P}_{\mathrm{p}}$.

Figure 7 - SEM cross-section and surface micrographs of the Ta thin films deposited by DOMS with a $\mathrm{P}_{\mathrm{p}}$ of a, b) $49 \mathrm{~kW}$; c, d) $66 \mathrm{~kW}$; e, f) $82 \mathrm{~kW}$; g, h) $105 \mathrm{~kW}$; i, j) $130 \mathrm{~kW}$.

Figure 8 - Representative AFM scans $(2 \mu \mathrm{m} \times 2 \mu \mathrm{m})$ showing the surface morphology of Ta thin films deposited by DOMS with a $\mathrm{P}_{\mathrm{p}}$ of a) $49 \mathrm{~kW}$; b) $66 \mathrm{~kW}$; c) $82 \mathrm{~kW}$; d) 105 kW; e) $130 \mathrm{~kW}$. 
Figure 9 - Surface roughness calculated from the AFM scans of the films deposited by DOMS with increase of $\mathrm{P}_{\mathrm{p}}$.

Figure 10 - Hardness and Young's modulus of the Ta thin films deposited by DOMS with the increase of $\mathrm{P}_{\mathrm{p}}$. 
a)

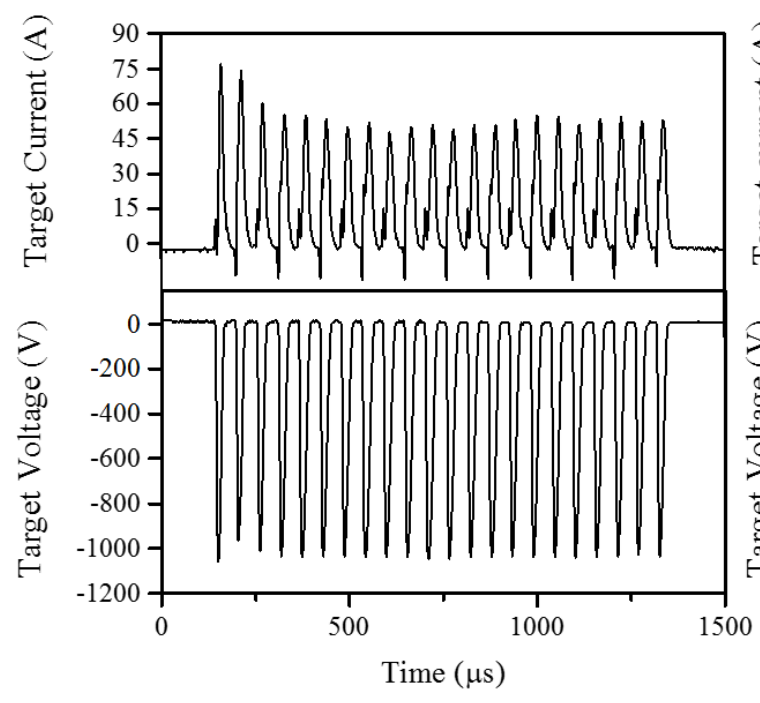

b)

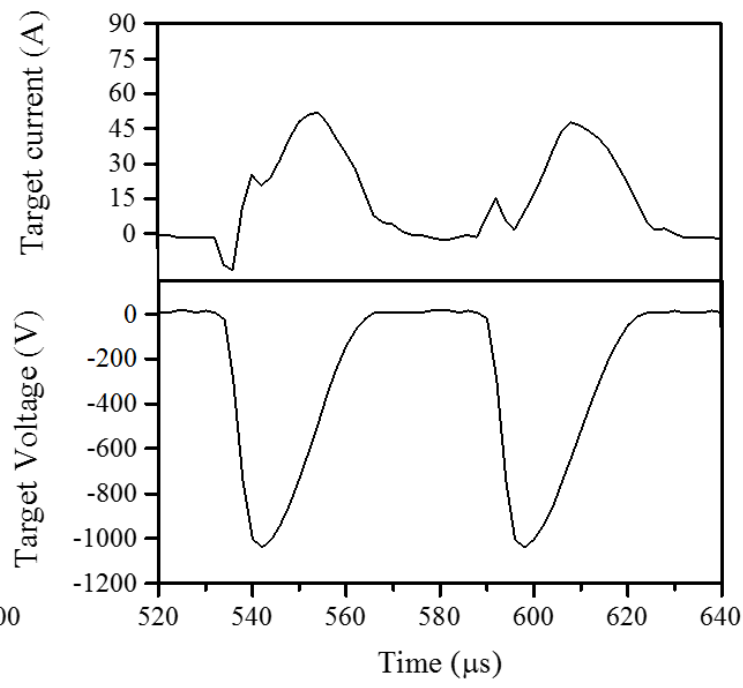


a)

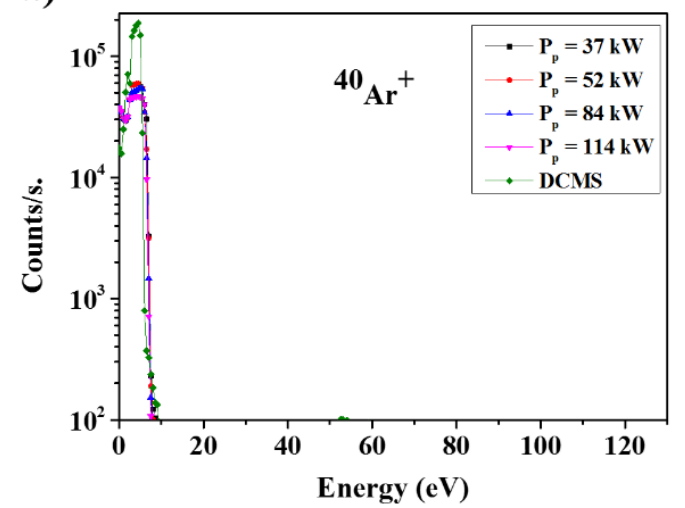

c)

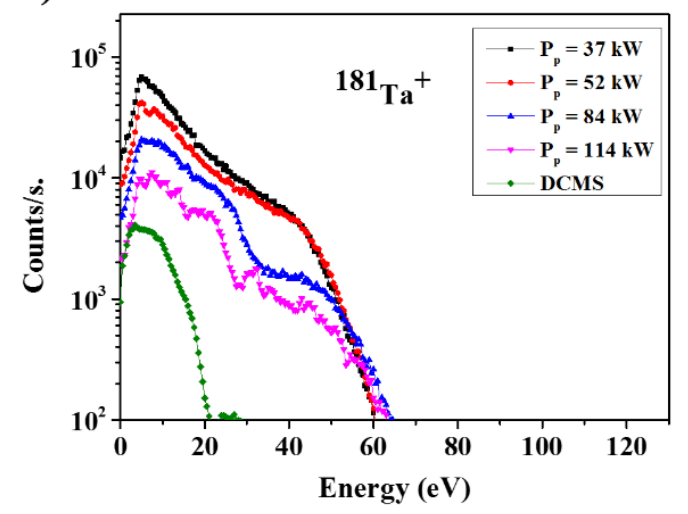

b)

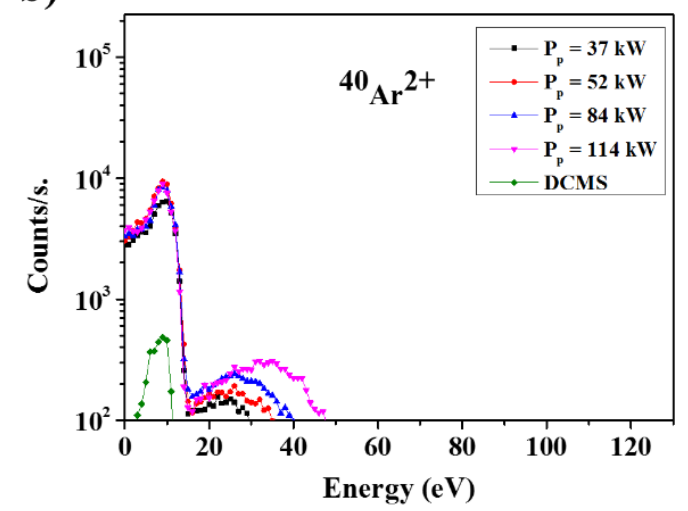

d)

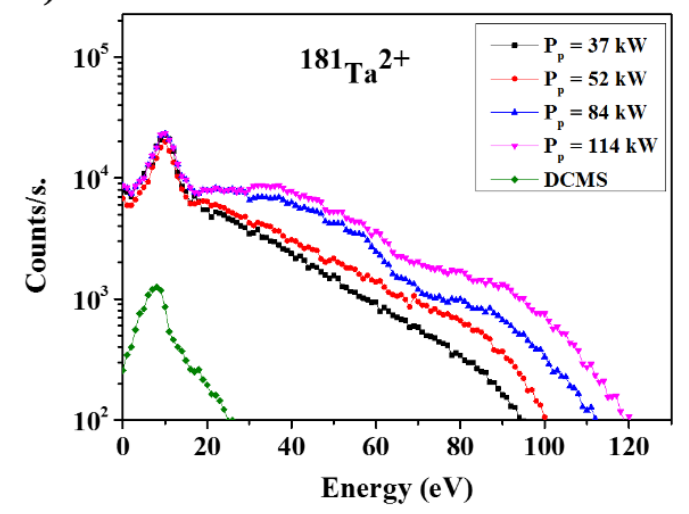




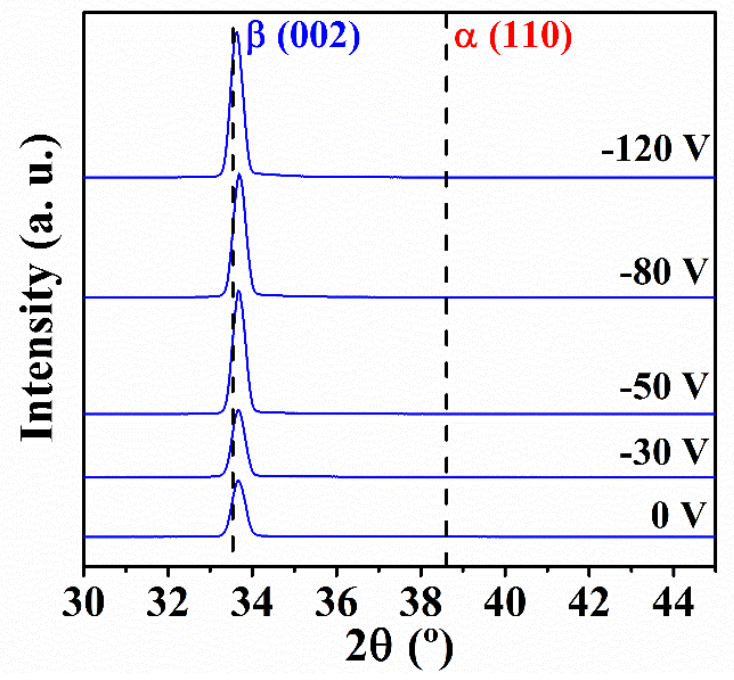

3 


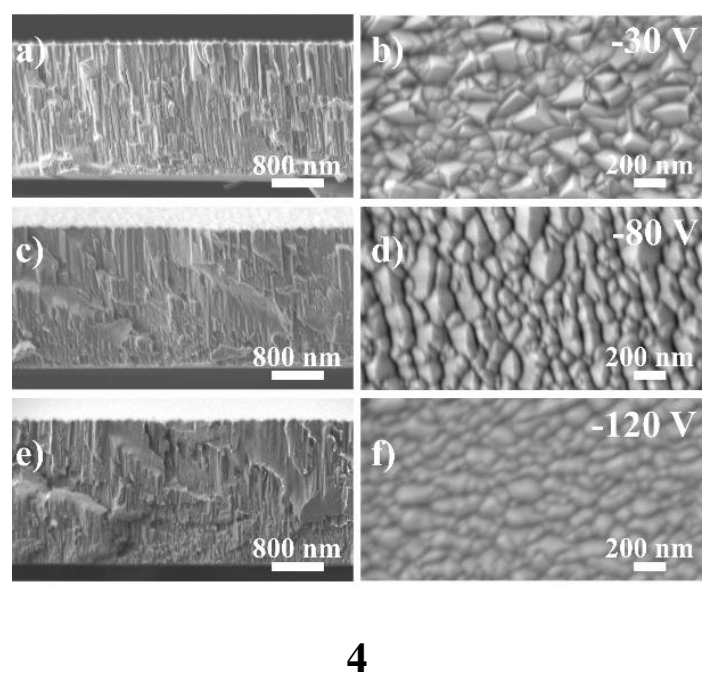




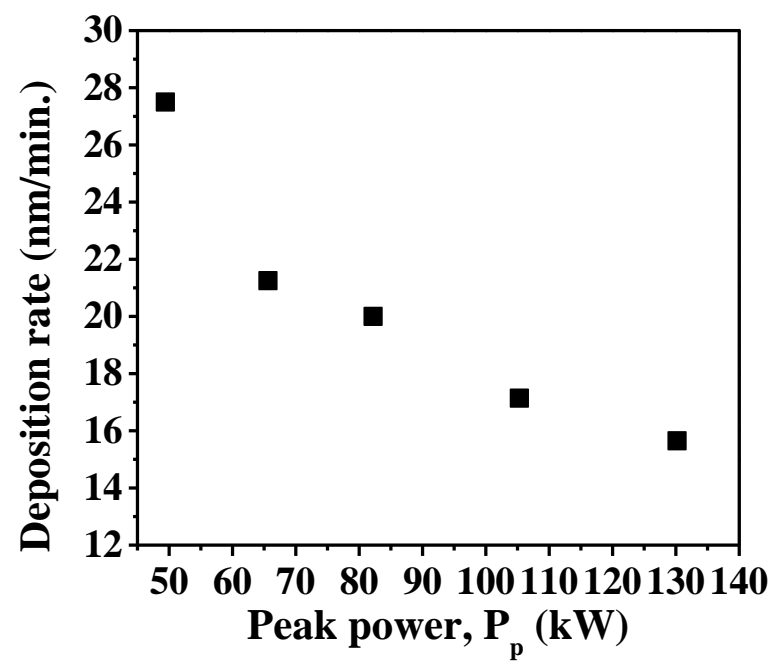

5 


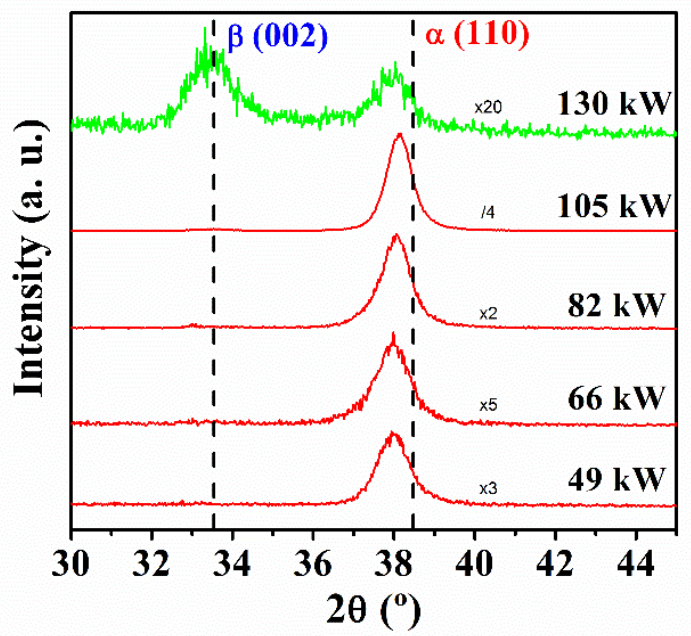

6 


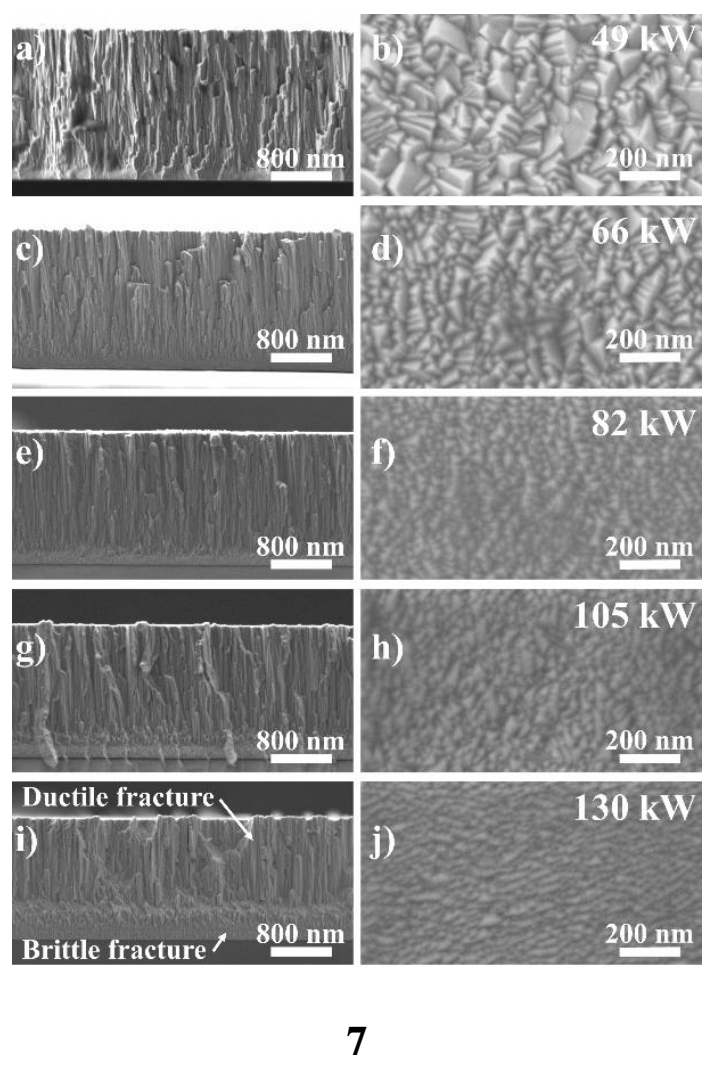



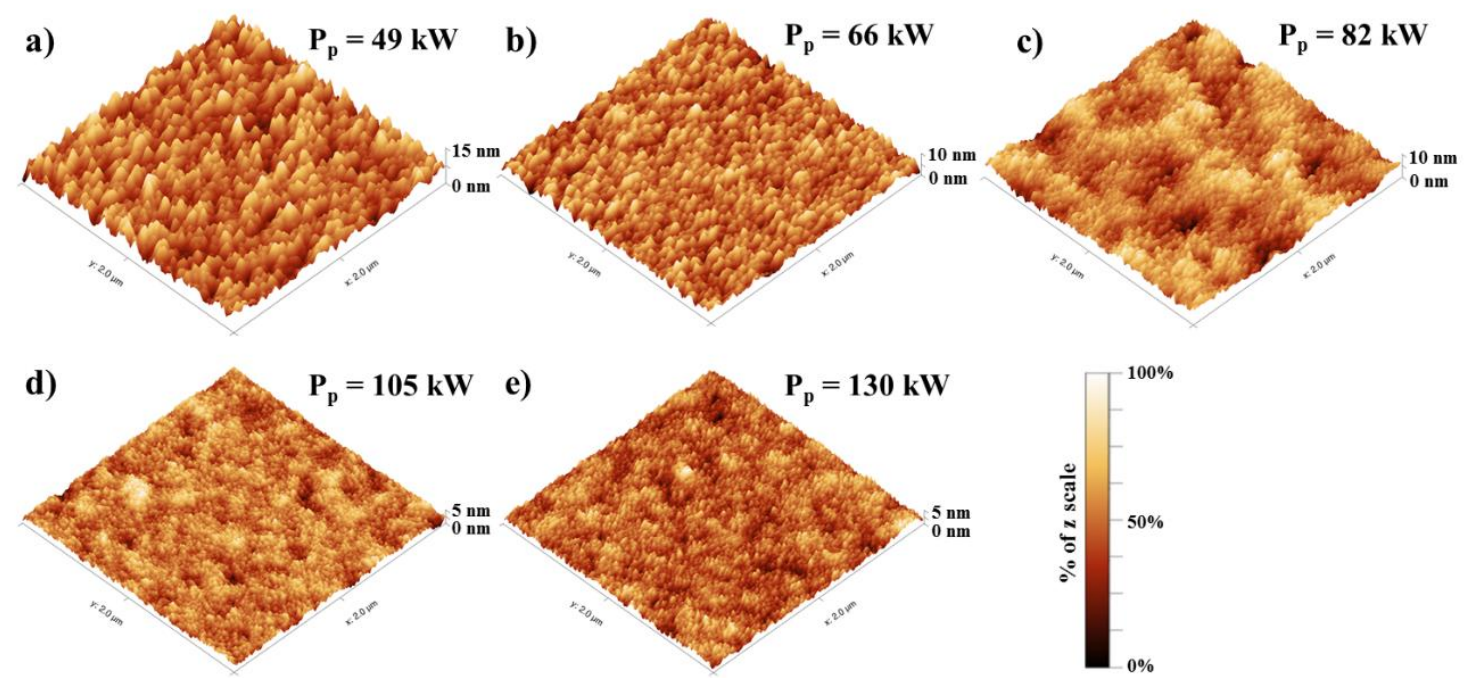


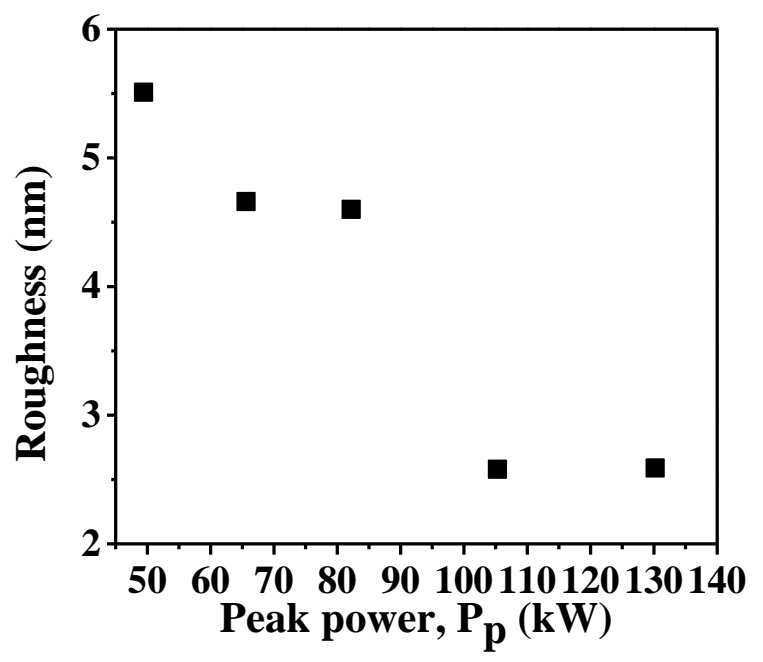

9 


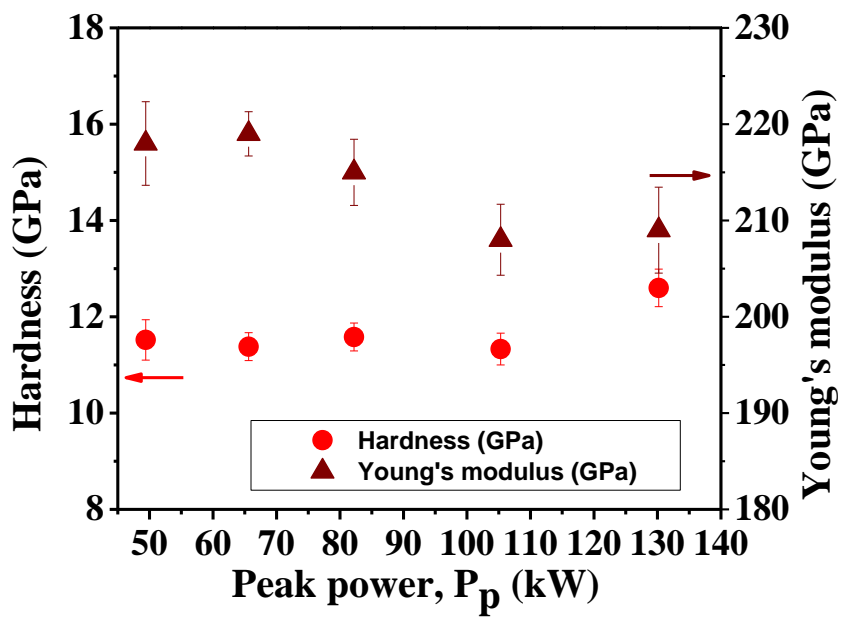

10 